\title{
Safety and effectiveness of eribulin in Japanese patients with locally advanced or metastatic breast cancer: a post-marketing observational study
}

\author{
Junichiro Watanabe ${ }^{1}$ - Yoshinori Ito $^{2} \cdot$ Shozo Ohsumi $^{3} \cdot$ Mitsuhiro Mizutani $^{4}$. \\ Hideya Tashiro ${ }^{5} \cdot$ Kenichi Sakurai $^{6}$ - Masato Takahashi ${ }^{7} \cdot$ Tsuyoshi Saito $^{8}$. \\ Junji Tsurutani $^{9}$ - Hirofumi Mukai ${ }^{10}$ - Tetsuhiro Yoshinami ${ }^{11} \cdot$ Shintaro Takao $^{12}$. $^{10}$ \\ Yasuhisa Yamamoto $^{13}$ - Toshiyuki Matsuoka ${ }^{14}$ - Hirotaka Iwase ${ }^{15}$ - Hiroji Iwata ${ }^{16}$. $^{\circ}$ \\ Seigo Nakamura ${ }^{17} \cdot$ Toshiaki Saeki $^{18}$
}

Received: 14 April 2017 / Accepted: 20 June 2017 /Published online: 29 June 2017

(C) The Author(s) 2017. This article is an open access publication

Summary Background This large-scale study was conducted to evaluate the safety and effectiveness of eribulin for the treatment of inoperable or recurrent breast cancer in real-

Electronic supplementary material The online version of this article (doi:10.1007/s10637-017-0486-4) contains supplementary material, which is available to authorized users.

Junichiro Watanabe

j.watanabe@scchr.jp

1 Division of Breast Oncology, Shizuoka Cancer Center, 1007 Shimonagakubo, Nagaizumi-cho, Sunto-gun, Shizuoka 411-8777, Japan

2 Department of Breast Medical Oncology, The Cancer Institute Hospital of Japanese Foundation for Cancer Research, 3-8-31, Ariake, Koto-ku, Tokyo 135-8550, Japan

3 Department of Breast Oncology, National Hospital Organization, Shikoku Cancer Center, 160, Minamiumemotomachikou, Matsuyamashi, Ehime 791-0280, Japan

4 Mikawa Breast Cancer Clinic, 39-6, Koita, Sasame-cho, Anjo, Aichi 446-0073, Japan

5 Department of Surgery, Oita Prefectural Hospital, 476, Bunyou, Oita, Oita 870-8511, Japan

6 Department of Breast Surgery, Nihon University School of Medicine, 30-1, Ohyaguchi Kami-cho, Itabashi-ku, Tokyo 173-8610, Japan

7 Department of Breast Surgery, National Hospital Organization, Hokkaido Cancer Center, 2-3-54, Kikusuishijo, Shiroishi-ku, Sapporo, Hokkaido 003-0804, Japan

8 Breast Surgery Unit, Japanese Red Cross Saitama Hospital, 1-5, Shintoshin, Chuo-ku, Saitama, Saitama 330-8553, Japan

9 Department of Medical Oncology, Kinki University Faculty of Medicine, 377-2, Onohigashi, Osaka-sayama, Osaka 589-8511, Japan world settings in Japan. Methods Between July and December 2011, eligible patients with inoperable or recurrent breast cancer receiving eribulin for the first time were
10 Division of Breast and Medical Oncology, National Cancer Center Hospital East, 6-5-1, Kashiwanoha, Kashiwa, Chiba 277-8577, Japan

11 Department of Clinical Oncology, Osaka Medical Center for Cancer and Cardiovascular Diseases, 1-3-3, Nakamichi, Higashinari-ku, Osaka, Osaka 537-8511, Japan

12 Department of Breast Surgery, Hyogo Cancer Center, 13-70, Kitaojicho, Akashi, Hyogo 673-8558, Japan

13 Department of Surgery, Oomoto Hospital, 1-1-5, Oomoto, Kita-ku, Okayama, Okayama 700-0924, Japan

14 Oncology PMS Section, Oncology Medical Department, Medical Division, Eisai Co., Ltd., 4-6-10 Koishikawa, Bunkyo-ku, Tokyo 112-8088, Japan

15 Department of Breast and Endocrine Surgery, Graduate School of Medical Sciences, Kumamoto University, 1-1-1 Honjo, Chuo-ku, Kumamoto, Kumamoto 860-8556, Japan

16 Division of Immunology, Aichi Cancer Center Research Institute, 1-1 Kanokoden, Chikusa-ku Nagoya, Aichi 464-8681, Japan

17 Division of Breast Surgical Oncology, Department of Surgery, Showa University, 1-5-8 Hatanodai, Shinagawa-ku, Tokyo 142-8555, Japan

18 Department of Breast Oncology, Saitama Medical University International Medical Center, 1397-1, Yamane, Hidaka, Saitama 350-1298, Japan 
centrally registered and observed for 1 year. Eribulin was administered intravenously $\left(1.4 \mathrm{mg} / \mathrm{m}^{2}\right)$ on days 1 and 8 of every 3 -week cycle. The primary endpoint was the frequency and intensity of adverse drug reactions (ADRs). Secondary endpoints included overall response rate (ORR) and time to treatment failure (TTF). Results Of 968 patients registered at 325 institutions, 951 and 671 were included in the safety and effectiveness analyses, respectively. In the safety population, ADRs were observed in 841 patients $(88.4 \%)$. The most common ( $\geq 15 \%$ incidence) were neutropenia $(66.6 \%)$, leukopenia $(62.4 \%)$, lymphopenia $(18.4 \%)$, and peripheral neuropathy (16.8\%). The most common grade $\geq 3$ ADRs ( $>5 \%$ incidence) were neutropenia $(59.8 \%)$, leukopenia $(50.5 \%)$, lymphopenia $(16.1 \%)$, and febrile neutropenia $(7.7 \%)$. In the effectiveness population, ORR was $16.5 \%$ (95\% confidence interval: 13.7 , 19.4). The median TTF was 127 days (95\% confidence interval: 120,134). Conclusions The safety and effectiveness profile of eribulin was consistent with prior studies. Eribulin had a favorable risk-benefit balance when used in real-world clinical settings.

Keywords Breast cancer · Eribulin · Japan · Post-marketing surveillance $\cdot$ Real world

\section{Introduction}

Breast cancer is the second most common cancer in the world and the most common cancer among women, with 1.67 million new cases diagnosed in 2012 [1]. In Japan, breast cancer was the sixth leading cause of death among women in 2014 $(20.6 / 100,000)$ [2]. Despite improvements in treatment, metastatic breast cancer (MBC) remains incurable and is the most common cause of death among patients with breast cancer [3, 4]; therefore, the goals of therapy are to prolong survival, palliate symptoms, and improve quality of life [5].

Anthracycline- and taxane-based regimens are currently the standard of care for adjuvant and first-line treatment for MBC. However, the long-term survival of patients with MBC remains poor. The 5-year survival of patients with stage IV breast cancer can be as low as $21 \%$, in comparison to $100 \%$ in patients with stage I breast cancer [6]. In Japan, 5- and 10-year relative survival rates for patients with stage IV breast cancer were as low as $32.6 \%$ and $15.6 \%$, respectively [7]. In addition, few options are available for treatment of patients with MBC who have been pre-treated with anthracyclines and taxanes, or those who have become resistant to anthracyclines and taxanes [3]. Thus, alternative treatment options that provide survival benefits for MBC patients are warranted.

Eribulin, a synthetic derivative of halichondrin B isolated from Halichondria okadai, is a new non-taxane microtubule dynamics inhibitor with a mechanism of action distinct from currently available taxanes. Unlike taxanes, eribulin binds to a single site on tubulin and to a small number of sites at microtubule ends [8]. Owing to its unique mechanism of action, eribulin displays antitumor activity in patients with welldefined taxane resistance [9].

Eribulin has received approval from the United States Food and Drug Administration and the European Medicines Agency for the treatment of locally advanced breast cancer and MBC refractory to both anthracyclines and taxanes [10]. EMBRACE, a randomized, phase III study in patients with heavily pre-treated $\mathrm{MBC}$, reported a significant and clinically meaningful improvement in overall survival (OS) in patients treated with eribulin (median OS, 13.1 months; 95\% confidence interval $[\mathrm{CI}]: 11.8,14.3)$ compared with those who received physician's choice of treatment (median OS, 10.6 months; 95\% CI: 9.3, 12.5; hazard ratio [HR], 0.81; 95\% CI: $0.66,0.99 ; p=0.041$ ) [11]. In another randomized, phase III study that included pre-treated patients with locally advanced breast cancer or MBC, median OS in the eribulin group was 15.9 months, compared with 14.5 months in the capecitabine group (HR, 0.88; 95\% CI: $0.771 .00 ; p=0.056)$ [12]. A pooled analysis of these two phase III studies demonstrated that eribulin significantly prolonged OS compared to the control (median OS, 15.2 months vs 12.8 months; HR, 0.85 ; 95\% CI: $0.77,0.95 ; p=0.003)$; OS data also favored eribulin in the various subgroups assessed [13]. Since toxicity does not increase during long-term treatment, eribulin can help maintain stable disease while providing high quality of life [14]. In addition, phase II studies have demonstrated the antitumor activity of eribulin with a manageable tolerability profile in extensively pre-treated patients who had previously received an anthracycline, taxane, and capecitabine [15-17].

In Japan, eribulin was approved for the treatment of inoperable or recurrent breast cancer in April 2011, following its approval in the United States (November 2010), Singapore (February 2011), and Europe (March 2011). However, the phase II eribulin study conducted in Japan included only 81 patients, and pre-marketing clinical studies included only a small number of Japanese patients [15]. Given the limited evidence of eribulin's safety and effectiveness, specifically in Japanese populations, our current observational study was conducted as a post-marketing commitment to the Ministry of Health, Labour and Welfare of Japan to assess the safety and effectiveness of eribulin in patients with inoperable or recurrent breast cancer in routine clinical settings in Japan.

\section{Methods}

\section{Study design}

This was a post-marketing, observational study conducted in 325 centers to evaluate the safety and effectiveness of eribulin mesylate (Halaven®, Eisai Co., Ltd., Japan) in Japanese 
patients with inoperable or recurrent breast cancer (ClinicalTrials.gov ID:NCT01463891). Patients were enrolled from July 19, 2011 (the launch date of Halaven®), to December 17, 2011, and were observed for 1 year following enrollment. Patients who discontinued treatment within 1 year were observed until the end of the cycle in which treatment was discontinued. Patients who continued treatment with eribulin for more than 1 year were observed through the treatment cycle ending at the 1 year mark. The study was conducted in accordance with the Declaration of Helsinki and Japanese regulatory requirements stipulated in Good Post-Marketing Study Practices (GPSP). Approval from the institutional ethics committee/institutional review board was obtained prior to commencement of the study. For this type of study formal consent was not required.

\section{Patients}

Patients with inoperable or recurrent breast cancer receiving treatment with eribulin for the first time were registered in this study by central registration. At institutions with study contracts specifying the number of patients to be registered, patients receiving their first treatment with eribulin were enrolled until this target number was reached. Patients with contraindications to treatment (high myelosuppression, known hypersensitivity to eribulin, pregnancy, or the possibility of pregnancy) were excluded [18].

\section{Treatment}

Eribulin was administered intravenously at a dose of $1.4 \mathrm{mg} /$ $\mathrm{m}^{2}$ on days 1 and 8 of every 3 -week cycle. Dosing was adjusted or discontinued depending on the condition of individual patients.

\section{Assessments}

The primary endpoint was the frequency and intensity of adverse drug reactions (ADRs). Safety was assessed throughout the study by recording adverse events (AEs). Severity and causality in relation to eribulin were assessed for each AE; when a causal relationship could not be ruled out, the $\mathrm{AE}$ was considered an ADR. ADRs that were not consistent with eribulin's prescribing information were considered unexpected. AEs and ADRs were graded according to the Japanese version of Common Terminology Criteria for Adverse Events (version 3.0) and tabulated using the Japanese version of the Medical Dictionary for Regulatory Activities (version 16.1).

Patients with hepatic function disorder were defined as those with aspartate aminotransferase (AST) or alanine aminotransferase (ALT) levels $>2.5$ times the upper limit of normal (ULN), or total bilirubin (T-Bil) levels $>1.5$ times the
ULN, before the start of eribulin. Patients whose pretreatment levels of AST, ALT, and T-Bil were unavailable were designated as having unknown hepatic function status. Patients with renal function disorder were defined as those with serum creatinine ( $\mathrm{SCr}$ ) levels $>1.5$ times the ULN before the start of eribulin. Patients whose pre-treatment $\mathrm{SCr}$ data were unavailable were classified as patients with unknown renal function status.

Secondary endpoints included overall response rate (ORR) and time to treatment failure (TTF). Effectiveness was evaluated using best overall response, as determined by individual physicians at each study center according to Response Evaluation Criteria in Solid Tumors version 1.1. Imaging techniques included computed tomography, magnetic resonance imaging, and X-rays. These evaluations were scheduled according to the clinical practice standards of each institution rather than by a protocol of fixed intervals. Response was classified as follows: complete response (CR), partial response $(\mathrm{PR})$, stable disease (SD), progressive disease (PD), and not evaluable (NE). ORR was defined as $\mathrm{CR}+\mathrm{PR}$; disease control rate (DCR) was defined as $\mathrm{CR}+\mathrm{PR}+\mathrm{SD}$; and clinical benefit rate $(\mathrm{CBR})$ was defined as $\mathrm{CR}+\mathrm{PR}+\mathrm{SD} \geq 6$ months. TTF was defined as the time from the first dose of eribulin until the date of treatment discontinuation from any cause (e.g., death, documentation of disease progression, adverse events, or patient's request), or was censored at the date of last follow-up for surviving patients remaining on treatment.

\section{Statistical analysis}

A sample size of 500 patients was estimated to be large enough to detect at least one case of severe infection (known frequency $0.5 \%$ ) at a probability of $90 \%$. Fisher's exact test was used for comparison between 2 groups, whereas a chisquare test was used for comparison among 3 or more groups. The Kaplan-Meier method was used to calculate TTF. All analyses were performed using Statistical Analysis System (SAS) Version 9.1.3 (SAS Institute Inc., Cary, NC, USA).

\section{Results}

\section{Patient disposition and baseline characteristics}

A total of 968 patients were registered at 325 institutions. The safety analysis included 951 patients; 17 were excluded due to a history of eribulin administration, refusal to fill in the case report forms, and lack of eribulin administration after registration. Of these, 671 patients were included in the effectiveness analysis; 280 were excluded due to a lack of diagnostic imaging data.

Baseline characteristics were similar between the safety and effectiveness populations (Table 1). In the safety 
Table 1 Patient demographics and baseline characteristics

\begin{tabular}{llll}
\hline $\begin{array}{l}\text { Safety analysis set } \\
n=951\end{array}$ & $\begin{array}{l}\text { Effectiveness analysis set } \\
n=671\end{array}$ \\
$n$ & $(\%)$ & $n$ & $(\%)$
\end{tabular}

\section{Gender}

Female

949

(99.8)

669

Male

$$
2
$$

(0.2)

2

(99.7)

Age (years)

$\begin{array}{lrlr}\leq 64 & 701 & (73.7) & 499 \\ 65-74 & 204 & (21.5) & 143 \\ \geq 75 & 46 & (4.8) & 29\end{array}$

Median (range)

$59.0(26-88)$

ECOG performance status

HER

481

$1 \quad 364$

2

3

4

HER 2/neu

Negative

Positive

Unknown

364

(50.6)

362

(38.3)

86

(9.0)

18

(1.9)

2

(0.2)

\section{$59.0(26-88)$}

$(74.4)$

(21.3)

(4.3)

ER

$\begin{array}{lrlrl}\text { Negative } & 285 & (30.0) & 186 & (27.7) \\ \text { Positive } & 641 & (67.4) & 472 & (70.3 \\ \text { Unknown } & 25 & (2.6) & 13 & (1.9) \\ \text { PgR } & & & & \end{array}$

$\begin{array}{llll}\text { Negative } & 444 & (46.7) & 304\end{array}$

$\begin{array}{lll}\text { Positive } & 472 \quad \text { (49.6) } & 347\end{array}$

Unknown

(3.7)

20

Triple negative

No 745

(78.3)

542

Yes

(18.4)

Unknown

(3.3)

110

19

Metastatic lesions

Breast $\quad 97 \quad(10.2)-65$

Lymph nodes $\quad 450$

(47.3)

65

332

Lung

(44.7)

305

(50.7) $\quad 344$

Liver

482

(55.8)

376

(12.4)

80

Brain

(16.3)

(17.4)

97

120

Others

165

$(84.4)$

580

(11.1) 63

(4.4) 28

Renal impairment ${ }^{\mathrm{b}}$

No

859

(90.3)

610

Yes

12
(90.9)

(53.9)

(37.4)

(7.6)

(0.9)

(0.1)

(73.8)

(18.5)

(7.7)

(27.7)

(70.3)

(45.3)

(51.7)

(80.8)

(16.4)

(9.7)

(49.5)

(45.5)

(51.3)

(56.0)

(11.9)

(14.5)

(17.9)

$(86.4)$

(1.2)
Table 1 (continued)

\begin{tabular}{|c|c|c|c|c|}
\hline & \multicolumn{2}{|c|}{$\begin{array}{l}\text { Safety analysis set } \\
n=951\end{array}$} & \multicolumn{2}{|c|}{$\begin{array}{l}\text { Effectiveness analysis set } \\
n=671\end{array}$} \\
\hline & $n$ & $(\%)$ & $n$ & $(\%)$ \\
\hline Unknown & 80 & $(8.4)$ & 53 & $(7.9)$ \\
\hline \multicolumn{5}{|c|}{ Number of previous chemotherapy regimens ${ }^{c}$} \\
\hline 0 & 41 & $(4.3)$ & 28 & $(4.2)$ \\
\hline 1 & 68 & $(7.2)$ & 51 & (7.6) \\
\hline 2 & 149 & $(15.7)$ & 108 & $(16.1)$ \\
\hline 3 & 161 & $(16.9)$ & 116 & $(17.3)$ \\
\hline 4 & 155 & $(16.3)$ & 109 & $(16.2)$ \\
\hline$\geq 5$ & 377 & $(39.6)$ & 259 & $(38.6)$ \\
\hline Median (range) & \multicolumn{2}{|c|}{$4.0(0-14)$} & \multicolumn{2}{|c|}{$4.0(0-14)$} \\
\hline
\end{tabular}

ECOG Eastern Cooperative Oncology Group, ER estrogen receptor, $H E R 2 / n e u$ human epidermal growth factor receptor type2, $P g R$ progesterone receptor

${ }^{\text {a }}$ Patients with hepatic function disorder were defined as those showing aspartate aminotransferase (AST) or alanine aminotransferase (ALT) levels of $>2.5$ times the upper limit of normal (ULN) or total bilirubin (T-Bil) levels of $>1.5$ times the ULN before the start of eribulin. Patients whose pre-treatment data were unavailable for AST, ALT, and T-Bil were counted as patients with unknown hepatic function status

${ }^{\mathrm{b}}$ Patients with renal function disorder were defined as those showing serum creatinine ( $\mathrm{SCr}$ ) levels of $>1.5$ times the ULN before the start of eribulin. Patients whose pre-treatment $\mathrm{SCr}$ data were unavailable were counted as patients with unknown renal function status

${ }^{\mathrm{c}}$ Chemotherapy for inoperable or recurrent breast cancer

population, median age of patients was 59.0 years (range, 26-88). The proportion of patients with human epidermal growth factor receptor type 2 (HER2)-positive status was $18.4 \%$ in the safety population and $18.5 \%$ in the effectiveness population; estrogen receptor (ER)-positive, $67.4 \%$ and $70.3 \%$; progesterone receptor (PgR)-positive, 49.6\% and $51.7 \%$; and triple negative, $18.4 \%$ and $16.4 \%$. The median number of previous chemotherapy regimens was 4.0 (range, $0-14$ ).

\section{Dose exposure}

In the safety population, $71.7 \%$ of patients received an initial eribulin dose of $1.4 \mathrm{mg} / \mathrm{m}^{2}, 19.1 \%$ received $1.1 \mathrm{mg} / \mathrm{m}^{2}$, and $3.8 \%$ received $0.7 \mathrm{mg} / \mathrm{m}^{2}$. Treatment lasted for a median of 4 cycles (range, 1-19), and median duration of exposure to eribulin was 14.1 weeks (range, 3-59). Median relative dose intensity was 0.75 (range, $0.21-1.25)$. Eribulin was administered concomitantly with chemotherapy, hormone therapy, or radiotherapy in $7.7 \%$, $16.3 \%$, and $5.8 \%$ of patients, respectively (Table 2 ). Trastuzumab was used in combination with eribulin in 
Table 2 Dose exposure to eribulin

\begin{tabular}{|c|c|c|c|c|}
\hline & \multicolumn{2}{|c|}{$\begin{array}{l}\text { Safety analysis set } \\
n=951\end{array}$} & \multicolumn{2}{|c|}{$\begin{array}{l}\text { Effectiveness analysis set } \\
n=671\end{array}$} \\
\hline & $n$ & $(\%)$ & $n$ & $(\%)$ \\
\hline \multicolumn{5}{|c|}{ Initial dose $\left(\mathrm{mg} / \mathrm{m}^{2}\right)$} \\
\hline 1.4 & 682 & $(71.7)$ & 489 & $(72.9)$ \\
\hline 1.1 & 182 & $(19.1)$ & 121 & $(18.0)$ \\
\hline 0.7 & 36 & $(3.8)$ & 24 & (3.6) \\
\hline Other $^{\mathrm{a}}$ & 51 & $(5.4)$ & 37 & $(5.5)$ \\
\hline \multicolumn{5}{|l|}{ Number of cycles } \\
\hline Median (range) & \multicolumn{2}{|c|}{$4.0(1-19)$} & \multicolumn{2}{|c|}{$5.0(1-19)$} \\
\hline \multicolumn{5}{|c|}{ Duration of exposure (weeks) } \\
\hline Median (range) & \multicolumn{2}{|c|}{$14.1(3-59)$} & \multicolumn{2}{|c|}{$18.0(3-59)$} \\
\hline \multicolumn{5}{|c|}{ Number of administrations (times) } \\
\hline Median (range) & \multicolumn{2}{|c|}{$8.0(1-36)$} & \multicolumn{2}{|c|}{$10.0(1-36)$} \\
\hline \multicolumn{5}{|c|}{ Relative dose intensity } \\
\hline Median (range) & \multicolumn{2}{|c|}{$0.750(0.21-1.25)$} & \multicolumn{2}{|c|}{$0.750(0.21-1.03)$} \\
\hline \multicolumn{5}{|c|}{ Concomitant chemotherapy } \\
\hline No & 877 & $(92.2)$ & 615 & $(91.7)$ \\
\hline Yes & 73 & $(7.7)$ & 56 & $(8.3)$ \\
\hline Unknown & 1 & $(0.1)$ & 0 & - \\
\hline \multicolumn{5}{|c|}{ Concomitant hormone therapy } \\
\hline No & 796 & $(83.7)$ & 544 & $(81.1)$ \\
\hline Yes & 155 & $(16.3)$ & 127 & $(18.9)$ \\
\hline \multicolumn{5}{|c|}{ Concomitant radiotherapy } \\
\hline No & 896 & $(94.2)$ & 629 & $(93.7)$ \\
\hline Yes & 55 & $(5.8)$ & 42 & $(6.3)$ \\
\hline
\end{tabular}

${ }^{\mathrm{a}}$ Initial dose of $0.8,0.9,1.0,1.1,1.2$, or $1.3 \mathrm{mg} / \mathrm{m}^{2}$
HER2-positive patients (34.3\%). Dose exposure was comparable in the effectiveness population.

\section{Safety analysis}

A total of 841 patients $(88.4 \%)$ reported ADRs. The most common ( $>10 \%$ incidence) ADRs observed were neutropenia (66.6\%), leukopenia (62.4\%), lymphopenia (18.4\%), peripheral neuropathy $(16.8 \%)$, alopecia $(12.1 \%)$, nausea (11.3\%), stomatitis (10.9\%), and pyrexia (10.3\%) (Table 3$)$. Grade $\geq 3$ ADRs with $>5 \%$ incidence were neutropenia (59.8\%), leukopenia $(50.5 \%)$, lymphopenia $(16.1 \%)$, and febrile neutropenia (7.7\%). ADRs leading to death were reported in 6 patients $(0.6 \%)$; these included pneumonia $(0.2 \%)$, liver metastasis $(0.1 \%)$, interstitial lung disease $(0.1 \%)$, and pulmonary bleeding $(0.1 \%)$, as well as sepsis $(0.1 \%)$, tumor lysis syndrome $(0.1 \%)$, and disseminated intravascular coagulation $(0.1 \%)$. Sepsis, tumor lysis syndrome, and disseminated intravascular coagulation developed in the same patient. ADRs leading to discontinuation of treatment with eribulin were reported in 93 patients $(9.8 \%)$; these included neutropenia $(2.3 \%)$, leukopenia $(2.3 \%)$, peripheral neuropathy (1.7\%), febrile neuropathy $(0.8 \%)$, lymphopenia $(0.7 \%)$, anorexia $(0.7 \%)$, thrombocytopenia $(0.6 \%)$, malaise $(0.6 \%)$, and interstitial lung disease $(0.5 \%)$.

\section{Effectiveness analysis}

CR and PR were observed in $1.3 \%$ and $15.2 \%$ of patients, respectively. The ORR was $16.5 \%$, DCR was $50.1 \%$, and CBR was $22.4 \%$ (Table 4). The median TTF was 127 days (95\% CI: 120, 134), with $7.9 \%$ censored cases (Fig. 1). The primary reasons for treatment discontinuation $(n=618)$ were disease progression $(86.1 \%)$, adverse events $(6.6 \%)$, patient's request $(3.7 \%)$, and death $(1.9 \%)$.

\section{Subanalysis}

Dose exposure

A subanalysis of initial eribulin dose by age group revealed that the proportion of patients receiving an initial dose of 
Table 3 Adverse drug reactions with an incidence higher than 5\% $(n=951)$

\begin{tabular}{lccccc}
\hline & \multicolumn{2}{l}{ All grade } & & \multirow{2}{l}{$\geq$ Grade 3} \\
\cline { 2 - 3 } \cline { 5 - 6 } & $n$ & $(\%)$ & & $N$ & $(\%)$ \\
\hline Overall & 841 & $(88.4)$ & 665 & $(69.9)$ \\
Hematologic events & & & & \\
Neutropenia & 633 & $(66.6)$ & 569 & $(59.8)$ \\
Leukopenia & 593 & $(62.4)$ & 480 & $(50.5)$ \\
Lymphopenia & 175 & $(18.4)$ & 153 & $(16.1)$ \\
Febrile neutropenia & 73 & $(7.7)$ & 73 & $(7.7)$ \\
Anemia & 63 & $(6.6)$ & 35 & $(3.7)$ \\
Non-hematologic events & & & & \\
Peripheral neuropathy & 160 & $(16.8)$ & 26 & $(2.7)$ \\
Alopecia & 115 & $(12.1)$ & & N/A \\
Nausea & 107 & $(11.3)$ & 4 & $(0.4)$ \\
Stomatitis & 104 & $(10.9)$ & 16 & $(1.7)$ \\
Pyrexia & 98 & $(10.3)$ & 2 & $(0.2)$ \\
Malaise & 93 & $(9.8)$ & 7 & $(0.7)$ \\
Decreased appetite & 81 & $(8.5)$ & 10 & $(1.1)$ \\
AST increased & 76 & $(8.0)$ & 15 & $(1.6)$ \\
Dysgeusia & 59 & $(6.2)$ & 0 & \\
ALT increased & 52 & $(5.5)$ & 15 & $(1.6)$ \\
C-reactive protein increased & 51 & $(5.4)$ & 6 & $(0.6)$ \\
\hline
\end{tabular}

$A S T$ aspartate aminotransferase, $A L T$ alanine aminotransferase, $N / A$ not available

$1.4 \mathrm{mg} / \mathrm{m}^{2}$ was smaller in patients aged $\geq 75$ years $(58.7 \%)$ than in patients aged $\leq 64$ years $(72.2 \%)$ or $65-74$ years (73.0\%). In addition, $52.8 \%$ of patients with hepatic dysfunction received an initial dose of $1.4 \mathrm{mg} / \mathrm{m}^{2}$, while $74.2 \%$ of patients without hepatic dysfunction received this initial dose (Online Resource 1).

\section{Safety analysis}

The incidence of ADRs of all grades in patients aged $\leq 64$ years, $65-74$ years, and $\geq 75$ years was $87.7 \%, 91.2 \%$, and $87.0 \%$, respectively. Grade $\geq 3$ ADRs in age groups $\leq 64$ years, $65-74$ years, and $\geq 75$ years occurred at an incidence of $70.2 \%, 68.1 \%$, and $73.9 \%$, respectively (Online Resource 2). Subanalysis of safety by hepatic function revealed that the incidence of ADRs of all grades was significantly higher $(p=0.0369)$ in patients with hepatic dysfunction (94.3\%) than in patients without hepatic dysfunction (87.4\%). Compared to patients without hepatic dysfunction, patients with hepatic dysfunction reported a higher incidence of thrombocytopenia ( $15.1 \%$ vs $2.1 \%$ ), febrile neutropenia ( $23.6 \%$ vs $5.7 \%$ ), and stomatitis (18.9\% vs $9.3 \%$ ) (Table 5 ).
Table 4 Effectiveness analysis $(n=671)$

$n$

$(\%)$

\begin{tabular}{lrrl} 
Best overall response & & \\
Complete response & 9 & $(1.3)$ \\
Partial response & 102 & $(15.2)$ \\
Stable disease & 225 & $(33.5)$ \\
Progressive disease & 330 & $(49.2)$ \\
$\quad$ Not evaluable & 5 & $(0.7)$ \\
Overall response rate $(\%)$ & 111 & $(16.5)$ \\
$\quad 95 \%$ CI $(\%)$ & & $(13.7,19.4)$ & \\
Disease control rate $(\%)$ & 336 & & $(50.1)$ \\
$\quad 95 \%$ CI $(\%)$ & & $(46.3,53.9)$ & \\
Clinical benefit rate $(\%)$ & 150 & & $(22.4)$ \\
$95 \%$ CI $(\%)$ & & $(19.2,25.5)$ & \\
\hline
\end{tabular}

CI confidence interval

\section{Effectiveness analysis}

The ORR of patients aged $\geq 75$ years $(34.5 \%)$ was significantly higher $(p=0.0290)$ than that of patients aged $\leq 64$ years $(15.8 \%)$ or $65-74$ years $(15.4 \%)$. The relatively small number of patients $\geq 75$ years $(n=46)$ suggests a possible bias; however, it is encouraging that older age was not associated with a lower ORR. In addition, the ORR of patients with hepatic dysfunction $(25.4 \%)$ was higher, although not significantly so $(p=0.0502)$, than that of patients without hepatic dysfunction $(15.5 \%)$. Patients receiving concomitant hormone therapy showed a significantly higher ORR $(p<0.001)$ than that of patients without hormone therapy ( $29.1 \%$ vs $13.6 \%)$. ORR was significantly lower in patients who had previously received a higher number of chemotherapy regimens ( 0 regimens, $35.7 \%$ vs $\geq 5$ regimens, $12.4 \% ; p=0.0069$ ) (Table 6 ).

\section{Discussion}

To our knowledge, this is the first large-scale, post-marketing observational study to examine the safety and effectiveness of eribulin in Japanese patients with inoperable or recurrent breast cancer in a real-world setting. Prior to this study, the only clinical research evaluating eribulin in Japanese patients with heavily pre-treated MBC was a phase II study of 81 patients [15]. By contrast, the current study included 951 patients and evaluated the safety and effectiveness of eribulin during its use in clinical practice.

The safety profile of eribulin in this study was largely consistent with the prior Japanese phase II study [15], with neutropenia, leukopenia, and lymphopenia as the most commonly reported ADRs. The current study reported ADRs rather than AEs as in the phase II study; however, there was a striking 
Fig. 1 Kaplan-Meier analysis of time to treatment failure in the effectiveness population. C.I. confidence interval

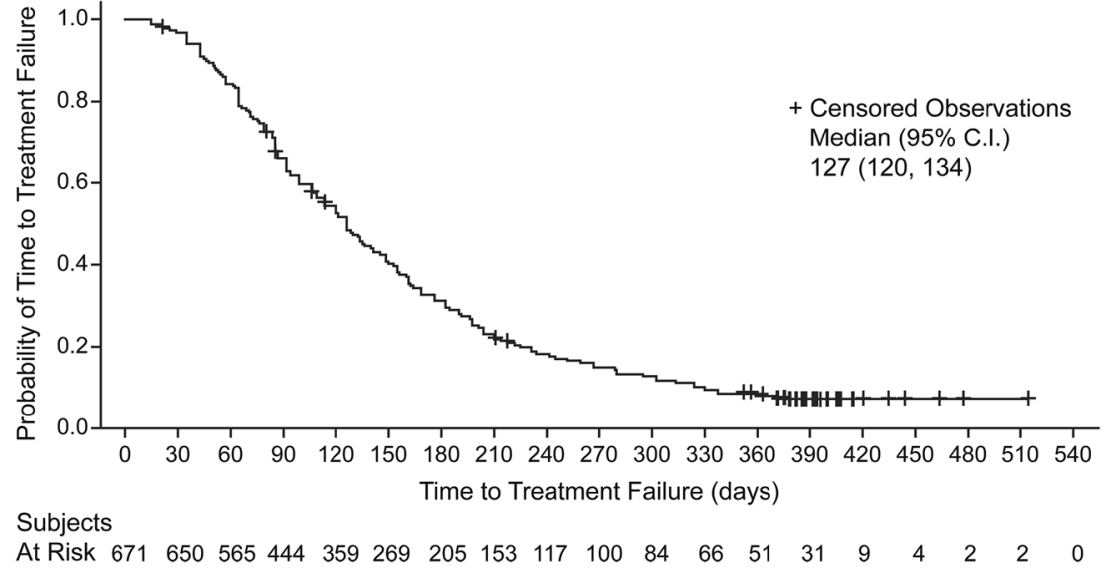

similarity between the most common ADRs and AEs in both studies. In the phase II study, the most frequently ( $\geq 50 \%$ incidence) occurring AEs included neutropenia (98.8\%), leukopenia $(98.8 \%)$, and lymphopenia $(54.3 \%)$ [15], whereas the most common ADRs ( $\geq 15 \%$ incidence) in the current study were neutropenia (66.6\%), leukopenia (62.4\%), and lymphopenia $(18.4 \%)$. These findings also match those of other phase II $[16,17]$ and phase III [12] studies showing hematological toxicities as the most common AE/ADR during treatment with eribulin, with neutropenia reported most frequently (50$65 \%$ ). Of note, grade $3 / 4$ neutropenia may be more pronounced in East Asian populations; the frequency of grade $3 / 4$ neutropenia in global trials was $20-65 \%$ [11, 12, 16, 17], compared to $85-95 \%$ in East Asian studies [15, 19]. Finally, the proportion of patients that discontinued treatment due to ADRs or AEs was $9.8 \%$ in the current study and $7.4 \%$ in the phase II study [15]. Therefore, the safety results from the current study corroborate the results from not only the previous phase II study in Japan, but other prior studies as well [11, 12, 16, 17].

Comparison of the incidence of ADRs among the three age groups ( $\leq 64$ years, $65-74$ years, and $\geq 75$ years) in this study revealed no significant differences among the groups. The proportion of patients receiving eribulin at an initial dose of $1.4 \mathrm{mg} /$ $\mathrm{m}^{2}$ was $72.2 \%, 73.0 \%$, and $58.7 \%$ in the three age groups, respectively, indicating that the initial dose was more frequently
Table 5 Subanalysis of common ( $\geq 10 \%$ incidence) adverse drug reactions by hepatic function

\begin{tabular}{|c|c|c|c|c|c|c|c|c|}
\hline & \multicolumn{4}{|c|}{$\begin{array}{l}\text { Without hepatic dysfunction } \\
n=803\end{array}$} & \multicolumn{4}{|c|}{$\begin{array}{l}\text { With hepatic dysfunction } \\
n=106\end{array}$} \\
\hline & \multicolumn{2}{|c|}{ All grades } & \multicolumn{2}{|c|}{$\geq$ Grade 3} & \multicolumn{2}{|c|}{ All grades } & \multicolumn{2}{|c|}{$\geq$ Grade 3} \\
\hline & $n$ & $(\%)$ & $n$ & $(\%)$ & $n$ & $(\%)$ & $n$ & $(\%)$ \\
\hline Overall & 702 & $(87.4)$ & 573 & (71.4) & 100 & $(94.3)$ & 92 & $(86.8)$ \\
\hline \multicolumn{9}{|l|}{ Hematologic events } \\
\hline Neutropenia & 533 & $(66.4)$ & 476 & $(59.3)$ & 70 & $(66.0)$ & 67 & $(63.2)$ \\
\hline Leukopenia & 494 & $(61.5)$ & 389 & $(48.4)$ & 75 & $(70.8)$ & 72 & $(67.9)$ \\
\hline Lymphopenia & 149 & (18.6) & 128 & $(15.9)$ & 23 & $(21.7)$ & 22 & $(20.8)$ \\
\hline Thrombocytopenia & 17 & $(2.1)$ & 8 & $(1.0)$ & 16 & $(15.1)$ & 9 & $(8.5)$ \\
\hline \multicolumn{9}{|l|}{ Non-hematologic events } \\
\hline Peripheral neuropathy & 136 & $(16.9)$ & 20 & $(2.5)$ & 12 & $(11.3)$ & 3 & $(2.8)$ \\
\hline Alopecia & 98 & $(12.2)$ & & N/A & 9 & $(8.5)$ & & $\mathrm{N} / \mathrm{A}$ \\
\hline Nausea & 89 & (11.1) & 2 & $(0.2)$ & 14 & $(13.2)$ & 2 & (1.9) \\
\hline Malaise & 81 & $(10.1)$ & 6 & $(0.7)$ & 5 & $(4.7)$ & 0 & \\
\hline Pyrexia & 79 & $(9.8)$ & 2 & $(0.2)$ & 12 & (11.3) & 0 & \\
\hline Stomatitis & 75 & $(9.3)$ & 9 & (1.1) & 20 & (18.9) & 6 & (5.7) \\
\hline Febrile neutropenia & 46 & $(5.7)$ & 46 & (5.7) & 25 & $(23.6)$ & 25 & (23.6) \\
\hline
\end{tabular}

N/A not available 
Table 6 Subanalysis of ORR by age, hepatic function, hormone therapy, and history of chemotherapy

\begin{tabular}{lccc}
\hline & $\begin{array}{l}\text { Total patients } \\
n\end{array}$ & $\begin{array}{l}\text { Patients achieving response } \\
n\end{array}$ & $\begin{array}{l}\text { ORR } \\
\%\end{array}$ \\
\hline $\begin{array}{l}\text { Age (years) } \\
\leq 64\end{array}$ & 499 & 79 & 15.8 \\
$65-74$ & 143 & 22 & 15.4 \\
$\geq 75$ & 29 & 10 & 34.5 \\
Hepatic dysfunction & & \\
No & 580 & 90 & 15.5 \\
Yes & 63 & 16 & 25.4 \\
Unknown & 28 & 5 & 17.9 \\
Concomitant hormone therapy & & \\
No & 544 & 74 & 13.6 \\
Yes & 127 & 37 & 29.1 \\
History of chemotherapy & & 35.7 \\
0 & 28 & 10 & 27.5 \\
1 & 51 & 14 & 13.9 \\
2 & 108 & 15 & 17.2 \\
3 & 116 & 20 & 18.3 \\
4 & 109 & 20 & 12.4 \\
$\geq 5$ & 259 & 32 & \\
\hline
\end{tabular}

$O R R$ overall response rate

adjusted for patients aged $\geq 75$ years but did not differ markedly between the $\leq 64$ years age group and the $65-74$ years age group (Online Resource 1). Taken together, these data suggest that reducing the initial dose from $1.4 \mathrm{mg} / \mathrm{m}^{2}$ to $1.1 \mathrm{mg} / \mathrm{m}^{2}$ or $0.7 \mathrm{mg} / \mathrm{m}^{2}$ for patients aged $\geq 75$ years may help avoid ADRs.

In a previous study analyzing the pharmacokinetic parameters of eribulin in patients with hepatic function classified using the Child-Pugh system (normal, mild dysfunction [Child-Pugh A], and moderate dysfunction [Child-Pugh B]), both mild and moderate hepatic dysfunction was associated with reduction of clearance, extension of half-life, increase of area under the curve (after correction for dose level), and increase of peak serum concentration (after correction for dose level) of eribulin [20]. Based on these findings, eribulin dose reduction should be recommended for patients with impaired hepatic function. In the current study, the proportion of patients receiving eribulin at an initial dose of $1.4 \mathrm{mg} / \mathrm{m}^{2}$ was $21.4 \%$ lower in patients with hepatic dysfunction than in patients without hepatic dysfunction (Online Resource 1). Therefore, although the initial dose of eribulin was adjusted for hepatic function, the incidence of ADRs was still higher in patients with hepatic dysfunction $(94.3 \%)$ than in patients without hepatic dysfunction (87.4\%). This indicates the need for evaluating hepatic function markers sufficiently before the start of eribulin treatment and considering initial dose reductions for patients with hepatic dysfunction.
The effectiveness of eribulin was evaluated using best overall response, as determined by diagnostic imaging conducted during the entire treatment period. It is difficult to directly compare results between the current study and the prior Japanese phase II study due to differences in tumor assessment methods (investigator review in this study versus independent review in the phase II study). However, the ORR of $16.5 \%$ in the current study $(95 \%$ CI $13.7,19.4)$ did not differ significantly from the ORR of $21.3 \%$ in the phase II study $(95 \%$ CI $12.9,31.8)$. In addition, response rate in the current study was higher than that of previous phase II [16, 17] and phase III [12] studies (approximately 9-14\%).

In the current study, the median TTF was 127 days. In prior phase II studies reported by Aogi et al. [15], Cortes et al. [16], and Vahdat et al. [17], median progression-free survival (PFS) was 3.7 months, 2.6 months, and 79 days, respectively. Median PFS in the eribulin group was 3.7 months in the randomized, phase III EMBRACE study [11]. In another randomized, phase III study [12], the median PFS in the eribulin group was 4.1 months. While caution is warranted when directly comparing TTF and PFS, TTF in the current study was longer than previously reported values of PFS from phase II [15-17] and phase III studies [11, 12]. In the current study, the interval between tumor assessments was not predetermined by protocol, but was instead performed according to the clinical practice standards of each institution; thus, TTF in the current study accurately reflects the utility of eribulin in the real-world setting.

Our analysis of factors affecting response to treatment indicated that ORR was higher in patients with hepatic dysfunction and patients receiving concomitant hormone therapy, and lower in patients that had previously received a large number of chemotherapy regimens for inoperable or recurrent breast cancer. As discussed previously, the initial dose of eribulin was reduced in patients with hepatic dysfunction; therefore, it is possible that the plasma concentration of eribulin was maintained at a steady state in these patients, resulting in higher effectiveness in patients with disturbed hepatic drug metabolism or excretion. Regarding the previous use of chemotherapy for inoperable or recurrent breast cancer, the prior Japanese phase II study also reported a decrease in ORR associated with an increase in the number of chemotherapy regimens $(36.0 \%$ for $0-1$ regimen, $14.7 \%$ for 2 regimens, and $14.3 \%$ for 3 regimens) comparable with ORRs reported in the current study (0 regimens, $35.7 \%, \geq 5$ regimens, $12.4 \%$; $p=0.0069$ ).

We identified two inherent limitations of this study. Of 951 patients included in this study (safety population), 671 patients were included in effectiveness analysis, and 280 patients were excluded due to a lack of diagnostic imaging data. Therefore, almost $30 \%$ of enrolled patients were not included in the effectiveness analysis. This can be interpreted as a reflection of real-world clinical practice 
where tumor response assessments may not be conducted routinely, and as a limitation of observational studies of chemotherapeutic agents. In addition, the study design and limited observation period (1 year) did not allow for sufficient survival analysis.

The results of this study showed safety and effectiveness profiles of eribulin similar to those seen in the pre-marketing clinical trial when used for patients with inoperable or recurrent breast cancer during clinical practice. Eribulin demonstrated a favorable risk-benefit balance when used in realworld clinical settings.

Acknowledgements This study was funded by Eisai Co., Ltd. (Tokyo, Japan). Writing, editorial, and graphics assistance was provided by Cactus Communications and sponsored by Eisai Co., Ltd. (Tokyo, Japan).

\section{Compliance with ethical standards}

Conflict of interest J. Watanabe is a medical advisor for Eisai Co., Ltd. and Astra Zeneca K.K. and has received honorarium from Eisai Co., Ltd., Kyowa Hakko Kirin Co., Ltd., AstraZeneca K.K., Taiho Pharmaceutical Co., Ltd., Novartis Pharma K.K., and Chugai Pharmaceutical Co., Ltd. Y. Ito received scholarship grants from Chugai Pharmaceutical Co., Ltd., Novartis Pharma K.K., PAREXEL International Inc., Eisai Co., Ltd., Taiho Pharmaceutical Co., Ltd., EPS Corporation, Daiichi Sankyo Co., Ltd., MSD K.K., Sanofi K.K., Eli Lilly Japan K.K., and AstraZeneca K.K. M. Takahashi received honoraria for lectures from AstraZeneca K.K., Chugai Pharmaceutical Co., Ltd., and Eisai Co., Ltd. T. Matsuoka is an employee of Eisai Co., Ltd. H. Iwata received honorarium from Eisai Co., Ltd. S. Nakamura is a medical advisor for Eisai Co., Ltd. (Tokyo, Japan). T. Saeki is a medical advisor for and received honorarium from Eisai Co., Ltd. All other authors have no conflicts of interest to declare.

Ethical approval The study was conducted in accordance with the Declaration of Helsinki and Japanese regulatory requirements stipulated in the GPSP. Approval from the institutional ethics committee/institutional review board was obtained prior to commencement of the study.

Informed consent For this type of study formal consent was not required.

Open Access This article is distributed under the terms of the Creative Commons Attribution 4.0 International License (http:// creativecommons.org/licenses/by/4.0/), which permits unrestricted use, distribution, and reproduction in any medium, provided you give appropriate credit to the original author(s) and the source, provide a link to the Creative Commons license, and indicate if changes were made.

\section{References}

1. World Health Organization, GLOBOCAN (2012) Breast cancer estimated incidence, mortality and prevalence worldwide in 2012. http://globocan.iarc.fr/old/FactSheets/cancers/breast-new.asp. Accessed 15 Mar 2017

2. National Cancer Center for Cancer Control and Information Services (2016) Japan cancer registry and statistics (in Japanese). http://ganjoho.jp/reg_stat/statistics/stat/summary.html. Accessed 15 Mar 2017

3. Cardoso F, Harbeck N, Fallowfield L, Kyriakides S, Senkus E, ESMO Guidelines Working Group (2012) Locally recurrent or metastatic breast cancer: ESMO clinical practice guidelines for diagnosis, treatment and follow-up. Ann Oncol 23(Suppl 7):viil1-vii19

4. Redig AJ, McAllister SS (2013) Breast cancer as a systemic disease: a view of metastasis. J Intern Med 274:113-126

5. Partridge AH, Rumble RB, Carey LA, Come SE, Davidson NE, Di Leo A et al (2014) Chemotherapy and targeted therapy for women with human epidermal growth factor receptor 2-negative (or unknown) advanced breast cancer: American society of clinical oncology clinical practice guideline. J Clin Oncol 32:3307-3329

6. Hayat MJ, Howlader N, Reichman ME, Edwards BK (2007) Cancer statistics, trends, and multiple primary cancer analyses from the surveillance, epidemiology, and end results (SEER) program. Oncologist 12:20-37

7. Ministry of Health, Labor and Welfare of Japan (2007) Survival rate surveillance (in Japanese). http:/www.gunma-cc.jp/sarukihan/ seizonritu/seizonritu2007.html. Accessed 15 Mar 2017

8. Smith JA, Wilson L, Azarenko O, Zhu X, Lewis BM, Littlefield BA et al (2010) Eribulin binds at microtubule ends to a single site on tubulin to suppress dynamic instability. Biochemistry 49:1331-1337

9. Inoue K, Saito T, Okubo K, Kimizuka K, Yamada H, Sakurai T et al (2016) Phase II clinical study of eribulin monotherapy in Japanese patients with metastatic breast cancer who had well-defined taxane resistance. Breast Cancer Res Treat 157:295-305

10. Crombag MR, Joerger M, Thürlimann B, Schellens JH, Beijnen JH, Huitema AD (2016) Pharmacokinetics of selected anticancer drugs in elderly cancer patients: focus on breast cancer. Cancers 8:6

11. Cortes J, O'Shaughnessy J, Loesch D, Blum JL, Vahdat LT, Petrakova $\mathrm{K}$ et al (2011) Eribulin monotherapy versus treatment of physician's choice in patients with metastatic breast cancer (EMBRACE): a phase 3 open-label randomised study. Lancet 377:914-923

12. Kaufman PA, Awada A, Twelves C, Yelle L, Perez EA, Velikova G et al (2015) Phase III open-label randomized study of eribulin mesylate versus capecitabine in patients with locally advanced or metastatic breast cancer previously treated with an anthracycline and a taxane. J Clin Oncol 33:594-601

13. Twelves C, Cortes J, Vahdat L, Olivo M, He Y, Kaufman PA et al (2014) Efficacy of eribulin in women with metastatic breast cancer: a pooled analysis of two phase 3 studies. Breast Cancer Res Treat 148:553-561

14. Cortes J, Hudgens S, Twelves C, Perez EA, Awada A, Yelle L et al (2015) Health-related quality of life in patients with locally advanced or metastatic breast cancer treated with eribulin mesylate or capecitabine in an open-label randomized phase 3 trial. Breast Cancer Res Treat 154:509-520

15. Aogi K, Iwata H, Masuda N, Mukai H, Yoshida M, Rai Y et al (2012) A phase II study of eribulin in Japanese patients with heavily pretreated metastatic breast cancer. Ann Oncol 23:1441-1448

16. Cortes J, Vahdat L, Blum JL, Twelves C, Campone M, Roché H et al (2010) Phase II study of the halichondrin B analog eribulin mesylate in patients with locally advanced or metastatic breast cancer previously treated with an anthracycline, a taxane, and capecitabine. J Clin Oncol 28:3922-3928

17. Vahdat LT, Pruitt B, Fabian C (2009) Phase II study of eribulin mesylate, a halichondrin $\mathrm{B}$ analog, in patients with metastatic breast cancer previously treated with an anthracycline and a taxane. J Clin Oncol 27:2954-2961

18. Halaven ${ }^{\circledR}$ Prescribing Information (for Japan, Version 6). http:// www.info.pmda.go.jp/go/pack/4291420A1022_1_06/. Accessed 15 Mar 2017

19. Park YH, Im YH, Lee KS, Park IH, Sohn J, Lee S et al (2015) Safety of eribulin in Korean patients with metastatic breast cancer. J Clin Oncol 33. doi:10.1200/jco.2015.33.15_suppl.e12031

20. Devriese LA, Witteveen PO, Marchetti S, Mergui-Roelvink M, Reyderman L, Wanders J et al (2012) Pharmacokinetics of eribulin mesylate in patients with solid tumors and hepatic impairment. Cancer Chemother Pharmacol 70:823-832 